\title{
Neutron imaging and tomography: Applications in food science
}

\author{
Camille Loupiac ${ }^{1,2, *}$, Simone Scussat ${ }^{1}$, Arnaud Hélary $^{2}$, Sylvain Desert ${ }^{2}$, and Frédéric Ott $^{2}$ \\ ${ }^{1}$ Equipe PCAV, UMRA 02.102 PAM, AgroSup Dijon, Université de Bourgogne-Franche Comté, 21000 Dijon, \\ France \\ ${ }^{2}$ Laboratoire Léon Brillouin, UMR 12 CEA - CNRS, CEA Saclay, 91191 Gif-sur-Yvette, France
}

\begin{abstract}
Neutron imaging and tomography allow observing the structure of large objects (from few to hundred centimeters) at a resolution around $100 \mu \mathrm{m}$. This nondestructive method is based on the detection of the transmission of a neutron beam through an object in 2D (imaging) or 3D after rotating the sample (tomography). The components of the object attenuate the neutron beam differently, depending on their composition, and produce accurate pictures of objects which give information about their structure. Neutron imaging has been used for quality control purposes in industries (aircraft, motor engineering...), but also find application from materials sciences (fuel cells, lithium batteries) through earth science (petrology, geology) to cultural heritage. More recently studies have been performed on food science topics.
\end{abstract}

\section{Introduction}

Neutron imaging and tomography are non - invasive and non - destructive methods which allow observing the structure of large objects (from few to hundred centimeters) at a resolution around $100 \mu \mathrm{m}$. Neutron imaging has been used for quality control purposes in industries that require precision machining such as aircraft or motor engineering [1-3]. Real-time imaging also allows following fast movement (few seconds) within objects, such as oil distribution or water uptake [4-7]. For example, today, one important application of neutron radiography is in testing the performance of fuel cells (in operando) by imaging water or hydrogen flow in the cells (in situ) [8-9]. Neutron imaging yields not only information on the quantity of the adsorbed water but also on its spatial distribution. Due to its high sensitivity to small amounts of hydrogenous compounds in a matrix, neutron radiography can be used to measured humidity transport, and is powerful for a number of applications- including studies of water propagation in heat exchangers and concrete structures, internal structure within geological samples, water uptake by plants, oil and fuel distribution in car engines and fuel injectors [1, 2, 3]. Neutron tomography has also been used in the cultural heritage studies and art world or in archeology to authenticate paintings and examine artifacts made of metal or stone [10-12]. Wood density is one of the most important wood characteristics for assaying wood quality as it provides an indication of the physical and mechanical properties of wood, such as strength, stiffness, hardness and hygroscopic properties $[13,14]$.

So, neutron imaging and tomography have been more and more used these last years to study and qualify the structure of materials in complex environments and more particularly their changes as

\footnotetext{
* Corresponding author: camille.loupiac@agrosupdijon.fr
} 
function of various parameters such as constraints, relative humidity, temperature, processing, or after storage etc. The size of the observed samples can varied from few millimeters to hundred centimeters. The resolution is around hundred micrometers. In the following text, we are going in a first part to introduce the principle of neutron imaging and the main characteristics of the existing instruments. Then, we will present a short overview of the results of the few studies performed on food science topics (food processing like drying and cooking, 3D microstructure of food matrices ....) or related fields (defects in packaging, hydration of root and plant leaves in agronomy...). We will show that food matrices can also be studied by neutron imaging and tomography during processing and over different conditions $(\mathrm{T}, \mathrm{P} \ldots)$.

\section{Principle and instruments}

\subsection{Principle}

Neutron imaging is based on the detection of the transmission of a neutron beam through an object. When this beam passes through the object components attenuate the neutron beam differently, depending on their composition, and produce accurate pictures of objects which give information about their structure. Like X-ray imaging, neutron imaging is made possible by the fact that different elements have different attenuation coefficients (Table 1). Hydrogen for example has a higher attenuation coefficient than lead or copper, which means that the signal detected for hydrogen will not be as strong as the signal detected for lead or copper. Moreover, the unique ability of neutron imaging to provide information on materials and structure, sometimes inside objects opaque to X-rays makes neutron radiography and tomography quite powerful. The contrast uniqueness in neutron imaging is based on the fact that neutrons interact with the nucleus inside the atom instead of electrons (as occurs with Xrays). This leads to a widely different contrast mechanism as compared to X-rays. Many organic materials are quite opaque to neutrons, whereas many metals are relatively transparent; hence they can be easily penetrated by neutrons. The interaction between the neutron beam and a material is generally described by the attenuation coefficient, which is a measure of the extent to which a material attenuates incident radiation. The attenuation coefficient (see Table 1) depends strongly on the elemental composition of the material and the actual neutron energy.

Table 1. Attenuation coefficients with thermal neutrons $\left(\mathrm{cm}^{-1}\right)[$ from 3].

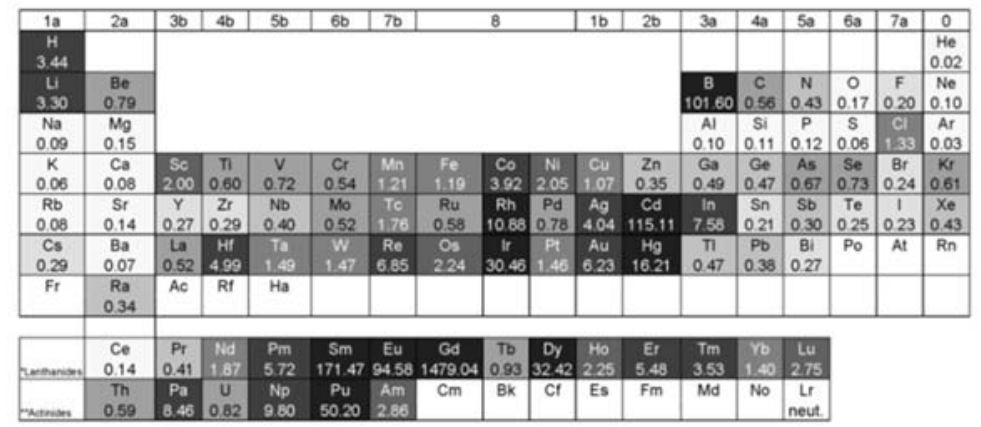

In accordance with the attenuation law, the intensity of the neutron beam behind the samples can be calculated as follows:

$$
I=I_{0} \times \mathrm{e}^{-(\Sigma \mathrm{d})}
$$


Where, $\mathrm{I}_{0}$ is the incident intensity of the neutron beam (grey levels); $\mathrm{I}$ is the weakened intensity of the neutron beam (grey levels); $\Sigma$ is the attenuation coefficient $\left(\mathrm{cm}^{-1}\right)$; $\mathrm{d}$ is the sample thickness $(\mathrm{cm})$.

Based on this equation, the attenuation coefficient $\Sigma$ can be calculated by:

$$
\Sigma=\ln \left(I_{0} / I\right) / d
$$

The attenuation coefficient is determined by the nuclear density $\mathrm{N}$ and the interaction probability $\sigma$ for the respective elements constituting the sample. However, the effective attenuation coefficient $\Sigma$ eff consists of the attenuation coefficients of the involved elements:

$$
\text { Leff } \quad=\quad \sum_{n}^{i=1} N_{i} \sigma_{i} \quad\left(\mathrm{~cm}^{-1}\right)
$$

Where $\mathrm{N}$ is the nuclear density (nuclei $/ \mathrm{cm}^{3}$ ) and $\sigma$ is the total cross section of interaction with the neutron of an element $\left(\mathrm{cm}^{2}\right)$

$$
\text { If } N i=\frac{\rho i}{M i} L\left(\text { nuclei } / \mathrm{cm}^{3}\right)
$$

Where $\rho$ is the density $\left(\mathrm{g} / \mathrm{cm}^{3}\right), \mathrm{L}$ is the Avogadro's constant and $\mathrm{M}$ is the atomic weight, the density of a sample can be evaluated through the following equation:

$$
\rho=\frac{\sum M}{L \sigma}\left(\mathrm{g} / \mathrm{cm}^{3}\right)
$$

For example, this equation (5) has been used to measure wood density and to compare the obtained results with the standard measurements realized by X-ray densitometry [13]. Density obtain with the two techniques are quite similar, and neutron brings several advantages, in particular its high sensitivity to hydrogen which allows more recently the visualization and quantification of liquid water in softwoods [14].

Transmission-based imaging using radiation could benefit to a great extent from the technical progress made in computing and data processing, allowing for the development and optimization of methods, measuring equipment, and research facilities. The increased brightness of modern neutron sources as well as pulsed nature of some of them, allow the improvement of the resolution of neutron tomography and radiography. The performance of detection devices (spatial and timing resolution) has to meet the challenges posed by those new imaging experiments in order to achieve the desired high resolution (around $10 \mu \mathrm{m}$ ), reduced the data acquisition times (few msec) and fully utilize the capabilities of modern neutron sources. Indeed, the improvement of spatial resolution in neutron imaging opens new fields of applications [4, 15-18].

\subsection{Instruments}

The main components of a neutron imaging instrument are presented in Figure 1 [16]. 


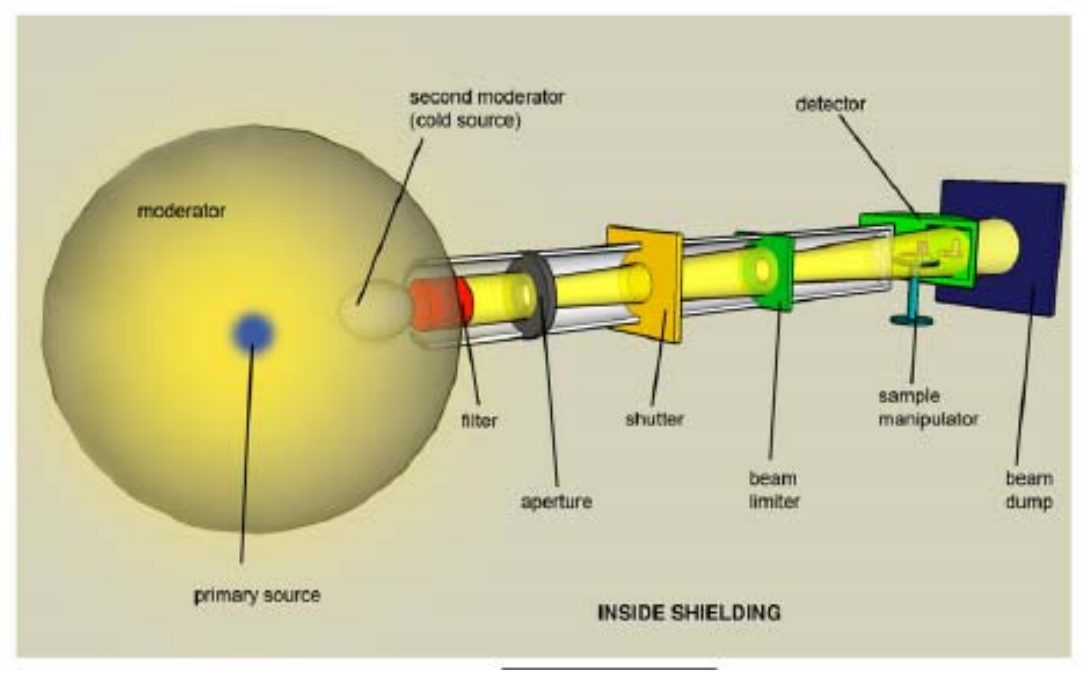

Figure 1. Schematic overview of a beamline design of a neutron imaging facility with the main components [from $16]$.

\subsubsection{Source: Cold versus thermal}

The cold neutron source allows different investigations compare to thermal ones. First the macroscopic cross-sections for materials are larger for cold neutrons due to the energy dependence of the absorption cross-section. The practical effect is that a higher contrast is achieved with cold neutrons due to energy dependence of the absorption cross-section.

\subsubsection{Beamline and collimation}

The initial neutrons from the source have to be tuned accordingly to get a well collimated beam of either thermal or cold neutrons. First of all, a moderator slows down the neutrons before a part of them can form the beam for imaging. This has to be done with apertures, filters, beam limiters and energy selectors. Most neutron imaging instruments are currently based on the simple pinhole geometry, in which the best achievable spatial resolution is limited by the $\mathrm{L} / \mathrm{D}$ ratio, where $\mathrm{L}$ is the distance between the "source" pinhole with a diameter $\mathrm{D}$ and the detector position. The beam size at the detector position is defined by the beam divergence and the distance L. In order to achieve higher spatial resolution, it is necessary to keep L/D high (by increasing L/decreasing D) and to position the sample as close as possible to the detector such that the image of the sample projected onto the detector can be considered to be formed by a parallel-beam that is perpendicular to the detection plane. The divergence of the beam (and therefore available beam size) at the detector position is thus also determined by the coating of the neutron guide. Most facilities possess two measuring positions at varying distances to the source and hence varying collimator lengths.

Kardjilov et al. [19] studied by simulation the impact of the neutron guide geometry on the image quality. Two focusing neutron guides (linearly tapered or cone-beam and elliptic) and a straight neutron guide with circular pinhole (for comparison) each of total length $3 \mathrm{~m}$ were simulated using Monte Carlo simulation code MCSTAS. The standard (straight guide with circular pinhole) configuration provides a beam that is just 1/9 the area of the beams produced by the focusing guides. Both experiments and simulations demonstrate superior spectral and geometry homogeneity of the cone-beam setup compared to the conventional pinhole geometry. The cone-beam geometry enables 
large beam cross section at relative short distances from the focal point. Use of the elliptic guide allowed enlargement of the beam dimensions by a factor of 3 at a distance of $5 \mathrm{~m}$ from the end of the guide to the detector at CONRAD (Berlin), thus enabling tomographic investigations of large samples. Results of Monte Carlo simulations as function of the different neutron guide configurations are presented in Figure 2.

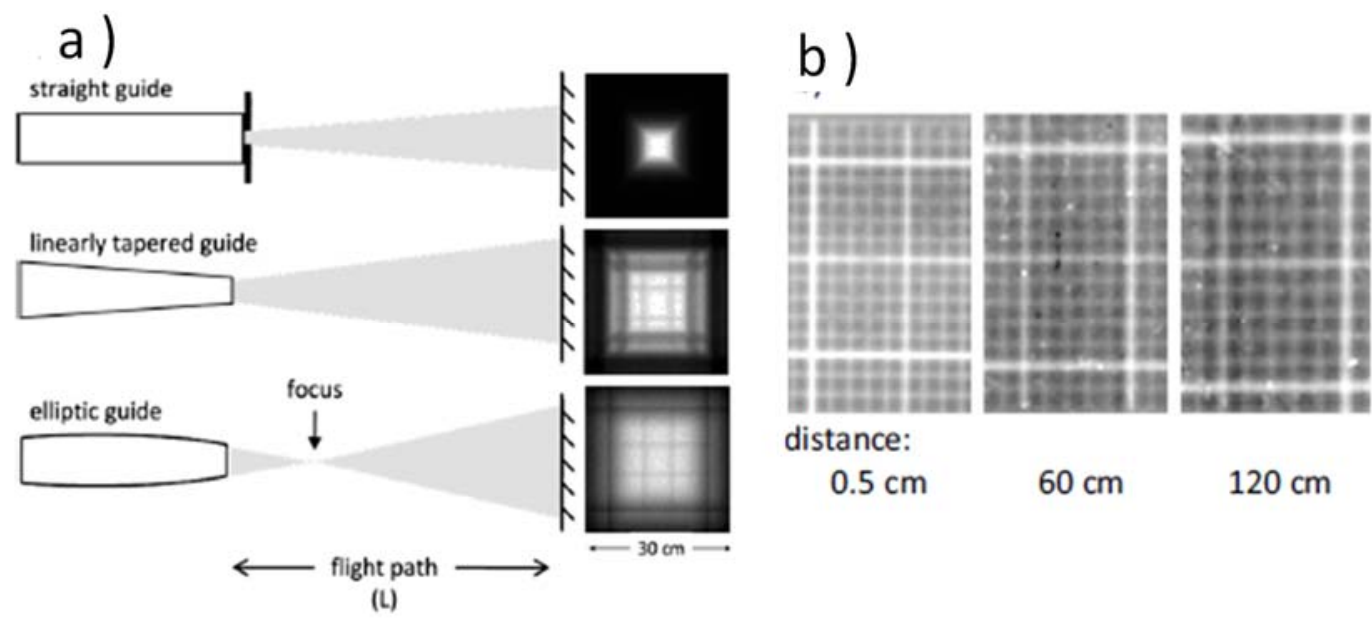

Figure 2. Impact of neutron guide geometry on image quality [From 19].

a) Monte Carlo simulations of the intensity distribution $5 \mathrm{~m}$ behind the guide exit in a detector plane of $30 \times 30 \mathrm{~cm}^{2}$ of different neutron guide: -straight guide with a pinhole of $1 \mathrm{~cm}$; - linearly tapered guide (coating $\mathrm{m}=3.0$, length: $3 \mathrm{~m}$, entrance: $3 \times 3 \mathrm{~cm}^{2}$, exit: $1 \times 1 \mathrm{~cm}^{2}$ ); - elliptic guide (coating $\mathrm{m}=3.0$, length: $3 \mathrm{~m}$, entrance: $3 \times 3 \mathrm{~cm}^{2}$, exit: $\left.0.89 \mathrm{x} 0.89 \mathrm{~cm}^{2}, \mathrm{~F} 1=7.5 \mathrm{~m}, \mathrm{~F} 2=0.2 \mathrm{~m}\right)$.

b) Imaging experiments showing the magnification of a grid with $1 \mathrm{~mm}$ periodicity measured at different distance from the detector in the cone-beam geometry.

\subsubsection{Detection}

The key component of a neutron imaging setup remains the imaging detector, a neutron sensitive twodimensional area placed perpendicular to the beam in order to observe the transmitted neutrons behind the object of investigations. A typical setup of the detection part of a neutron imaging instrument is shown in Figure 3. 


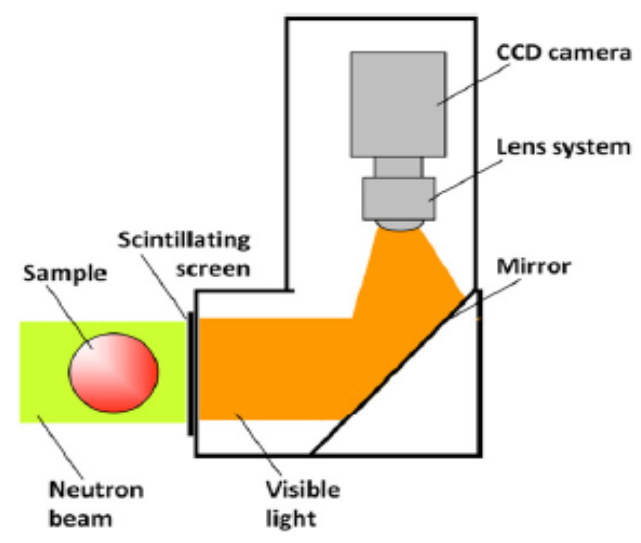

Figure 3. Detector setup for neutron imaging based on a CCD camera [from 19].

Among the detector systems used, the most common and highly flexible digital ones is based on cooled CCD cameras which are optimized for very high light sensitivity and long exposure times. The primary neutron sensitive detector is a scintillating screen (based on Lithium-6 or Gadolinium converters) which is observed by the CCD via a suitable lens system. To avoid any direct exposure of the sensitive electronics of the camera the emitted scintillation light is mirrored by $90^{\circ}$ or $180^{\circ}$.

\subsubsection{Scintillators}

Neutron detection is a more complicated task than the registration of ionizing particles. The neutron detection process is not possible in a direct manner because neutrons do not carry a charge which is needed for ionisation. Therefore a nuclear excitation reaction is needed, which can be absorption, collision or even fission. To enable a sensitive response, conversion materials with a high interaction cross-section are commonly used: Gd, B-10, Li-6, He-3 or U-235. The neutron capture results in gamma-, beta-radiation or the emission of charged particles, which initiate light emission in suitable materials, electronic excitation or other measurable signals (Koerner et al., 2000 ;Lehmann et al., 2004). A common used screen is made of $\mathrm{LiZnS}(\mathrm{Ag})$. LiZnS has relatively low neutron capture from Li-6 but offers high output (photons/neutron) from photon emission of $\mathrm{ZnS}$. Gadox $\left(\mathrm{Gd}_{2} \mathrm{O}_{2} \mathrm{~S}\right)$ exhibits much higher neutron capture from the very high cross section of gadolinium, with $90 \%$ absorption in a layer of $10 \mu \mathrm{m}$, but also a significantly lower light output. The free-mean-path length of conversion electrons (Resolution $=12 \mu \mathrm{m}$ ) is an order of magnitude less than the range of free-mean path length of triton particle in Li-6 (Resolution $=130 \mu \mathrm{m})$. The disadvantage of gadox scintillating screens in neutron imaging is their sensitivity to X-rays and the low light due to low energy produced.

The first step towards developing a high resolution detector system is the improvement of scintillating screen. The thickness of the scintillating screen significantly influences the resolution. Thinner scintillating layers improve the spatial resolution but decrease the efficiency, which leads to much longer exposure times. A step forward was the production of thin $\mathrm{LiZnS(Ag)} \mathrm{scintillating} \mathrm{screens}$ going down to $50 \mu \mathrm{m}$ layer thickness. The response of different scintillator screens has been studied by Kardjilov et al. [18] and the optimization of scintillators thickness and composition is resumed in Table 2.

Table 2. Spatial resolution of different scintillator screens: impact of thickness and composition [18].

\begin{tabular}{|l|l|l|l|}
\hline Scintillator & Thickness $(\mu \mathrm{m})$ & Exposure Time $(\mathrm{s})$ & Resolution $(\mu \mathrm{m})$ \\
\hline
\end{tabular}




\begin{tabular}{|l|l|l|l|}
\hline Lithium & 400 & $1 \times 20$ & 192 \\
\hline " & 200 & $1 \times 20$ & 137 \\
\hline “ & 100 & $1 \times 20$ & 108 \\
\hline Gadox & 50 & $1 \times 20$ & 72 \\
\hline "“ & 30 & $3 \times 20$ & 76 \\
\hline " & 20 & $3 \times 20$ & 57 \\
\hline
\end{tabular}

As reported in this table, a slightly better resolution is obtained with the $50 \mu \mathrm{m}$ lithium scintillator compared to the $30 \mu \mathrm{m}$ gadolinium one. This is due to the high level of noise observe with the latter. However, $50 \mu \mathrm{m}$ is the lowest thicknesses which can be obtained with lithium. Another key component of the detection system allowing to increase the quality of the resolution and which has been studied by Kardjilov et al. [19] is the optical lens associated to the camera. Indeed as can be seen in Table 3, by using lenses from $105 \mathrm{~mm}$ to $200 \mathrm{~mm}$ with same scintillator screens (for example $5 \mu \mathrm{m}$ ) the resolution obtained goes from $48 \mu \mathrm{m}$ to $25 \mu \mathrm{m}$.

Table 3. Conjunction of different optical lenses and various gadox scintillator thicknesses [19].

\begin{tabular}{|l|l|l|l|}
\hline Scintillator / lens & Thickness $(\mu \mathrm{m})$ & Exposure time $(\mathrm{s})$ & Resolution $(\mu \mathrm{m})$ \\
\hline Gadox /105 mm lens & 30 & $3 \times 20$ & 76 \\
\hline “ & 5 & $3 \times 20$ & 48 \\
\hline Gadox / 200 mm lens & 30 & $3 \times 30$ & 61 \\
\hline “ & 5 & $3 \times 30$ & 25 \\
\hline
\end{tabular}

Other ways to improve the resolution of the detection has been reported by Boillat et al. [15] and were typically designed for applications like fuel cell imaging. Indeed in this specific case, the resolution requirement has to be obtained in different directions (observation of the membrane of the cell orientated in position parallel and / or perpendicular to the neutron beam), such making anisotropic setups attractive. In a first step, the resolution was increased by using the detector in a tilted position as illustrated in Figure 4. The effect of the angle ( $\alpha$ with $90^{\circ}$ as value for the normal non tilted configuration) on the resolution was studied and an improvement of the resolution is observed. The resolution of $8.7 \mu \mathrm{m}$ obtained with a tilting angle of $3^{\circ}$ is probably still the highest resolution reported for such neutron imaging setup.

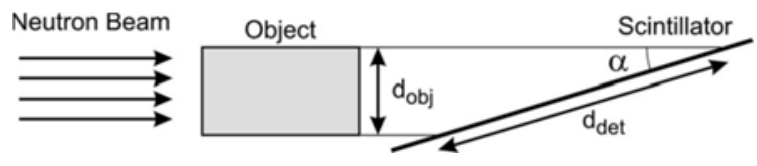

Figure 4. Principle of detector tilting $(\alpha)$. A feature of size of the object $\left(\mathrm{d}_{\mathrm{obj}}\right)$ is projected on a larger distance $\mathrm{d}_{\mathrm{det}}$ in the detector plane [15].

However a strong limitation for the resolution comes from the distance (Ld) between the sample and the detector. Also the tilting of detector may result in an increase in the Ld value, around $60 \mathrm{~mm}$ in a first setup which was decrease to this study to $25 \mathrm{~mm}$ by using an optical adapter which allowed placement of the scintillator in a tilted position without rotating the detector box.

Finally, by using a large source diameter and by increasing the L/D ratio specifically in one direction using beam limiters (Figure 5), the negative effect on the neutron flux can be mitigated. 


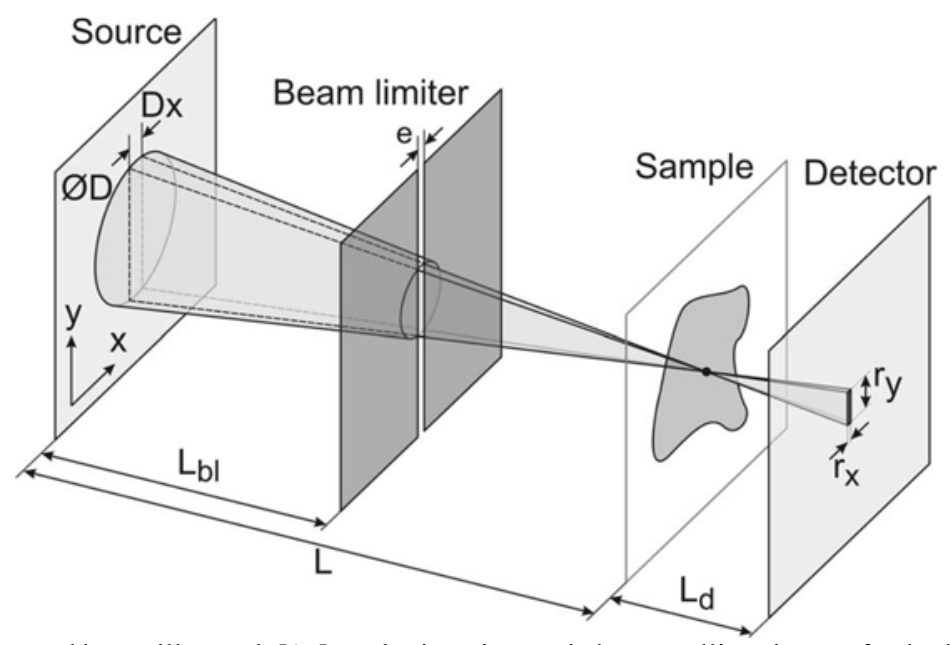

Figure 5. Geometry used by Boillat et al. [15] to obtain anisotropic beam collimation perfectly dedicated to through and in plane measurements.

Moreover, the requirement of spatial resolution for in plane imaging for example for fuel cell water migration can be perfectly attended with this configuration. Table 4, resumes the different parameters used in this study to perform such experiments and to increase the resolution.

Table 4. Typical setup parameters for in-plane imaging of fuel cells.

\begin{tabular}{|l|l|l|}
\hline Parameter & Anisotropic & Isotropic \\
\hline sample-detector distance & $25 \mathrm{~mm}$ & $25 \mathrm{~mm}$ \\
\hline L/Dx & 1200 & 1200 \\
\hline L/Dy & 89 & 1200 \\
\hline Tilting angle & $10^{\circ}$ & $10^{\circ}$ \\
\hline Neutron flux & $1.13 \times 10^{7}$ & $0.84 \times 10^{6}$ \\
\hline Horizontal resolution & 20 & 20 \\
\hline Vertical resolution & 200 & 50 \\
\hline Exposure time & $10 \mathrm{~s}$ & $2 \mathrm{~min}$ \\
\hline
\end{tabular}

To measure the obtained resolution as function of the tilting angles, radiograms of a test object consisting of a gadolinium pattern on a silicon substrate were acquired and are presented in Figure 6. The test object is a grid of perpendicular lines, but the feature used for the measurement of the resolution is a single line with a width of $150 \mu \mathrm{m}$. As observed on Figure 6A, some distortion of the object are introduced which has been attributed to the slightly bended surface of the detector. As shown in the same figure, these distortions can be corrected in a post processing step. 
A

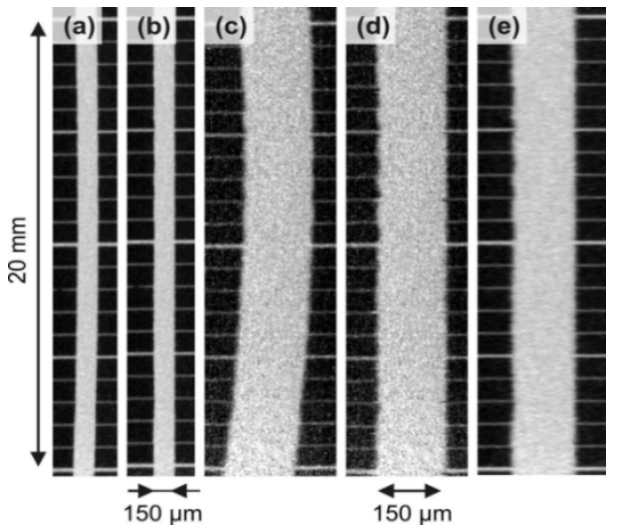

B

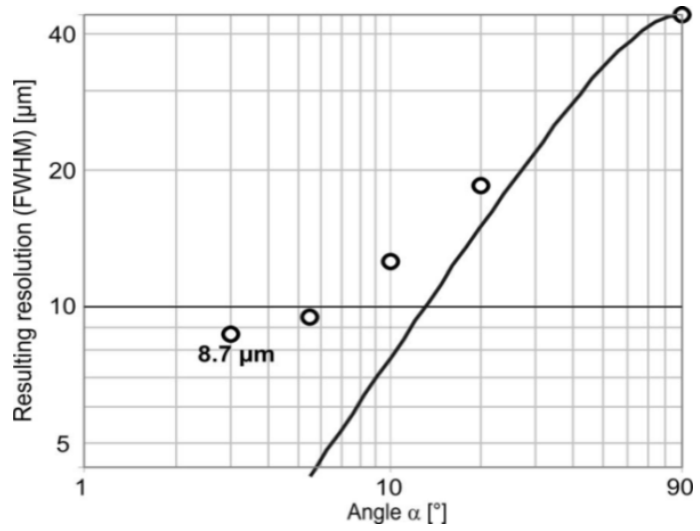

Figure 6. Radiograms of a test object consisting of a gadolinium pattern on a silicon substrate [15].

A- Images of $150 \mu \mathrm{m}$ wide test object. a) $\alpha=10^{\circ}$, b) $\alpha=10^{\circ}$ corrected, c) $\alpha=3$, d) $\alpha=3^{\circ}$ corrected, and e) same image as (b) magnified for comparison with (d).

B- Measured horizontal resolution as function of the tilting angle $\alpha$. The solid line represents the expected resolution as expected by the following equation: $\mathrm{reff}_{\mathrm{f}}=\mathrm{r} \operatorname{det} \mathrm{X} \sin \alpha$

The resolution of $8.7 \mu \mathrm{m}$ obtained with a tilting angle of $3^{\circ}$ is the highest resolution reported for neutron imaging.

This tilting geometry has been used by Boillat et al. [15] in the determination of liquid water in operating polymer electrolyte fuel cells (PEFCs). Figure 7 presented the evolution of the water distribution measured in the cathode side of an operating cell $\left(70^{\circ} \mathrm{C}, 2 \mathrm{bar}, 1 \mathrm{~A} / \mathrm{cm}^{2}\right)$.

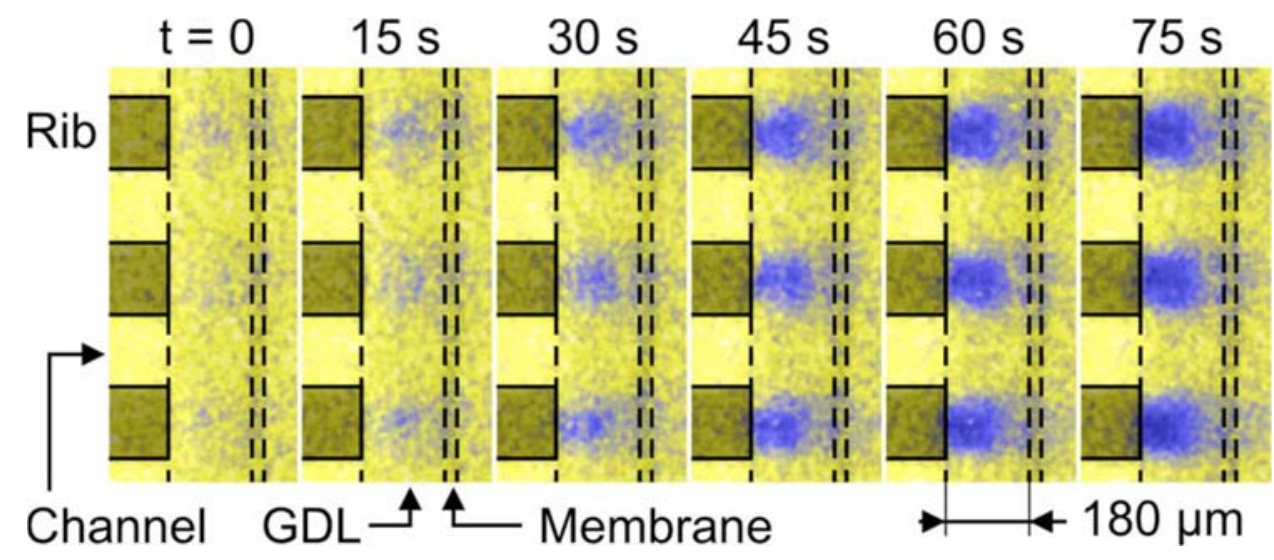

Figure 7. Evolution of the water distribution measured in the cathode (GDL) of an operating PEFC. Cathode gas flow is dry. Anode relative humidity is changed from 40 to $100 \%$ at $t=0$. Due to the magnifying effect of the tilted detector, the horizontal and vertical scales are not identical. The cell had an active area of $0.5 \mathrm{~cm}^{2} \mathrm{with}$ a depth of $5 \mathrm{~mm}$ in the beam direction [15].

The combination of spatial and temporal resolutions is clearly sufficient to observe the behavior of the water distribution during such a transient state. In particular, it can be observed that liquid water first appears in the depth of the gas diffusion layer before condensing in the layer just around the membrane. 
The layer just next to the membrane corresponding to the microporous layer exhibits a significantly lower content of liquid water.

\subsubsection{New detector: Microchannel plates}

Neutron counting detectors with MicroChannel Plates (MCP) provide some unique capabilities for high resolution applications [20-22]. MCPs have been widely used for electron/ion/photon detection, however neutron detection with 10B and/or Gd- enriched MCPs is quite new technology. Standard MCPs are basically a glass disk ( $\sim 1 \mathrm{~mm}$ thick) with densely packed cylindrical pores, each microscopic in dimension, typically ranging from 5 to $10 \mu \mathrm{m}$ diameter. Each hole operates as an independent electron multiplier, providing high-resolution 2-D imaging with a spatial resolution which is channel pitched limited, typically 7 to $12 \mu \mathrm{m}$ between adjacent pores. The process of neutron detection by the MCP consists of 3 stages: absorption of incoming neutron within the MCP glass, escape of the reaction products into adjacent pore, release of charged particles form the pore walls which initiate the electron avalanche in the pore [23]. Detectors with MCPs were measured to have efficiency levels as high as 70 $\%$ for cold neutrons, sub-15 $\mu \mathrm{m}$ spatial resolution and a $1 \mu \mathrm{s}$ timing resolution. Many different types of readout approaches exist for MCP detectors, one of them is the Medipix2/Timepix readout. One of the disadvantages of this system is the small active area $\left(14 \times 14 \mathrm{~mm}^{2}\right.$ per one chip).

\subsubsection{Resolution: Definition and measurement}

There are 3 competing demands in neutron imaging to optimize for each detection system and its practical use: spatial resolution, time resolution and image quality. As previously explained, the spatial resolution is not only defined by the detector but also by the beam properties. However with a beam collimation ratio L/D better than 500, the resulting image will be only influenced by the detector performance, when a small distance between the object and the detector plane can be realized. The time resolution is mainly given by the detector efficiency, meaning how fast an image can be produced in the sensitive area. This is the exposure time, texp, where neutrons are applied and converted into detectable signal. The time to download the image information to a permanent storage facility (development or readout time, tread) is influencing the time resolution too. The real frame rate is then defined as FR $=1 /$ (texp +tread). The image quality can be described in the first order by the signal-tonoise ratio of the image $(\mathrm{S} / \mathrm{N})$, which increases usually with longer exposure time. Therefore, a wide dynamic range is to prefer, typically 16 bits and more. The noise level is given by 3 contributions, the statistics level of the detection process itself (shot noise), that from the readout process (readout noise) and from the background effects as dark current or random effects from background radiation (dark noise). Spatial resolution is also related to the image quality because an image with high noise level is inherently blurred too.

The spatial resolving power of an image-forming system is the ability to separate image of two neighboring objects points. Various statements of the resolution have been given in the literature, including the pixel size of the detector, the thickness of the scintillator, or observability of small holes [24-26]. The Modulation Transfer Function (MTF) is a common metric used to quantify the spatial resolution. Methodology used to measure it, is described by the ISO 12233 standard [24]. The initial task in MTF measurement is the identification of suitable edges for the analysis. The edge must be oriented with a minimum tilt angle and this is made by selecting a rectangular region of interest (ROI) on a specific target (example gives in Figure 8a). For example, the region over which the calculations are done is defined as shown in Figure 8b. The Edge Spread Function (ESF) represents the pixel response in terms of gray levels along a line(s) perpendicular to the edge measured on an opaque object with a straight line (see Figure $8 \mathrm{~b}$ and $8 \mathrm{c}$ ). 

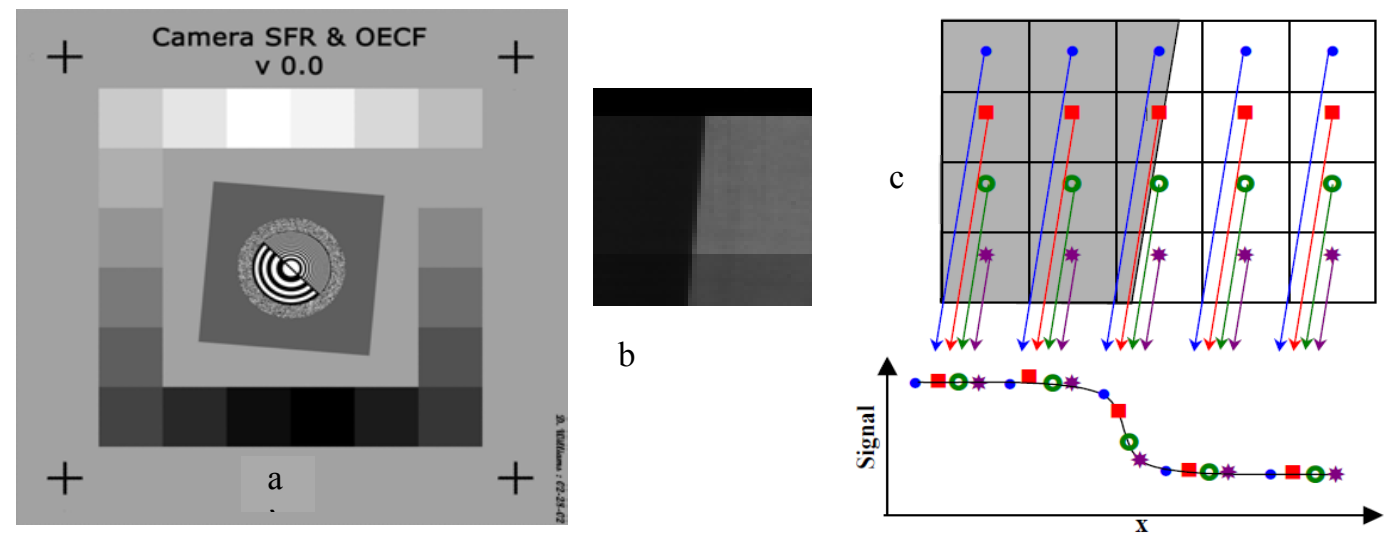

Figure 8. Resolution a) Edge prototype object, b) Selected Region of interest, c) Projection of data along the edge [24].

Edge-gradient analysis is a line spread function (LSF) transformation technique that derives the LSF. The term can be summarized as "the lost of contrast caused by the imaging system at any given spatial frequency". Rather than a simple spatial resolution limit, the imaging capabilities are fully described across the entire spatial frequency range (usually measured in the line pairs per millimeters, $1 \mathrm{p} / \mathrm{mm}$ ). An MTF curve clearly depicts that the finer detail, the lower contrast, highlighting the inter-related nature of resolution and contrast $[25,26]$.

Grunzweig et al [27] described their works on the fabrication and the application of a novel neutron imaging test device made of gadolinium and specifically designed for a real time evaluation of the spatial resolution of a neutron imaging detector. The developed test device consists of two geometrical patterns, a Siemens star and a square of grid lines (see Figure 9). The diameter of the Siemens star is $20 \mathrm{~mm}$ and consisted of 128 spokes. The period of the inner spoke is $40 \mu \mathrm{m}$ ( 25 line pairs mm-1) and the outer one is $500 \mu \mathrm{m}$ (2 line pairs mm-1). Concentric radial markers are at periods of $50,100,200,300,400$ and $500 \mu \mathrm{m}$. The second test pattern is a square of grid lines. Its size is $24 \mathrm{x}$ $24 \mathrm{~mm} 2$. The distance between two adjacent grid lines is $1 \mathrm{~mm}$. The grid lines have linewidths of 50, 100 , and $150 \mu \mathrm{m}$.
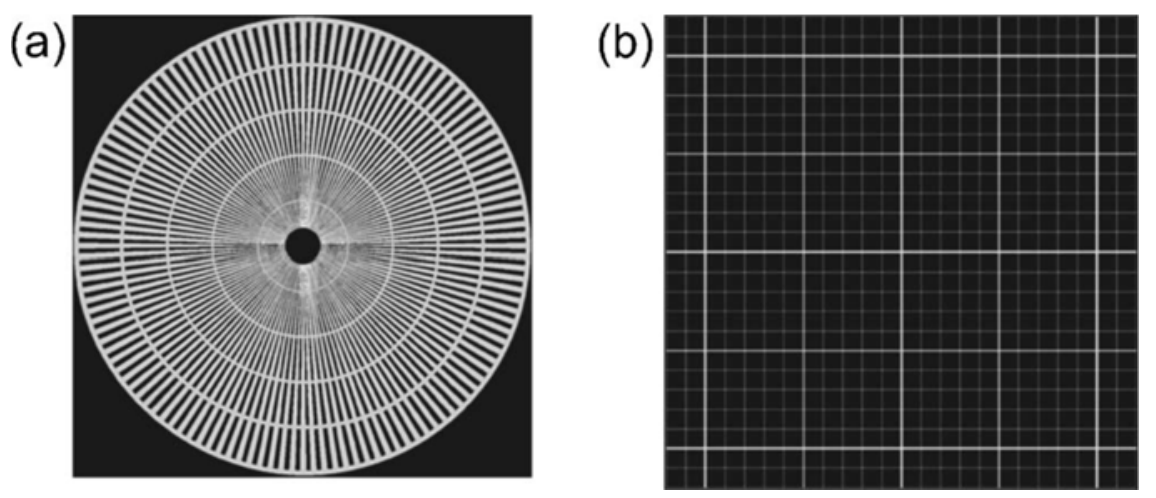

Figure 9. Geometrical patterns of the test device dedicated to the characterization of the detector performance. (a) Geometry of the Siemens star which is ideal for identifying the resolution limit in all directions. (b) Geometry of the square of grid lines which is designed to provide a convenient means for measuring distortions [27]. 


\section{Applications in food science}

Neutron imaging and tomography are excellent tools for evaluating water diffusion and uptake inside composite structure. These imaging methods were able to locate small quantities of water. For example, in food science, extrusion, drying and heating processing are technology commonly used. These processes are often applied in food and pharmaceutical industries for affecting product microstructure, product chemistry or the macroscopic shape of products. The texture of manufactured foods is related to their mechanical properties which in turn depend on their structure and the way it has been generated during processing. This is also the case for solid food foams, among which cereal products are particularly relevant. Microstructural characteristics such as cell size heterogeneity, macroporosity can be determined by neutron imaging. Moreover stability over storage at different relative humidity and how this storage can affect the porosity are key parameters that still need to be better characterized and understood. The problems observed at the macro-scale have prompted interest in understanding the processes going on a smaller scale, the pore scale for example. The intention is to gain an understanding of the fundamental pore-scale processes and thereby to be able to understand and describe the governing macro-scale processes. These topics, processing-structure-hydration can be covered by imaging measurements and the following part of this review will present some recent studies performed on food matrices.

\subsection{Imaging during processing}

\subsubsection{Drying fish}

Among food, the dry products allow to have a shelf life longer than liquid products, at room temperature and often under a reduced volume facilitating their packaging and transport. The drying of food is a process still studied today. The main aim is to decrease the development of microorganisms or to stop some chemical degradation by reducing the availability of the water. To reduce water content as low as possible in food matrices, two main drying processes are used mainly the spray-drying and for high value products the freeze drying. Such processes with different steps involving low or high temperatures and vacuum can deteriorate the structure and the organoleptic properties of the final products. Moreover, after drying, the ageing of the powders as function of the storage conditions need to be control as water sorption by the powders or the low moisture products can increase the mobility of the ingredients and of the water. This increase of mobility and water availability over storage can again provoke some physical change in the matrices and be detrimental for the final properties (texture, solubility, oxidation, flavor...) of the products. In this context, there is still a strong need of better understanding and characterization of the water repartition and mobility in food products during drying steps but also during storage at specific relative humidity. Neutron imaging and tomography have been used to analyze drying process of fish [28]. Different type of fish (mackerel and squid) involving different steps before drying (as previous salting steps for example), or different weights (small, middle and large) have been dried. Water losing during process was analyzed through the neutron beam. The analysis of the neutron image recorded on these samples showed that the loss of water was not homogeneously distributed among the fish. To have a better characterization on the spatial distribution of water during drying, tomography was also performed on mackerel dried by conventional process (soft breeze) and freeze dried. Some results are presented in Figure 10. In the freeze dried fish the repartition of remaining water seems to be more homogeneous along the overall fish than in the classically dried sample. Whatever the drying process, it was shown that in some part of head or organs, water content was kept high. 

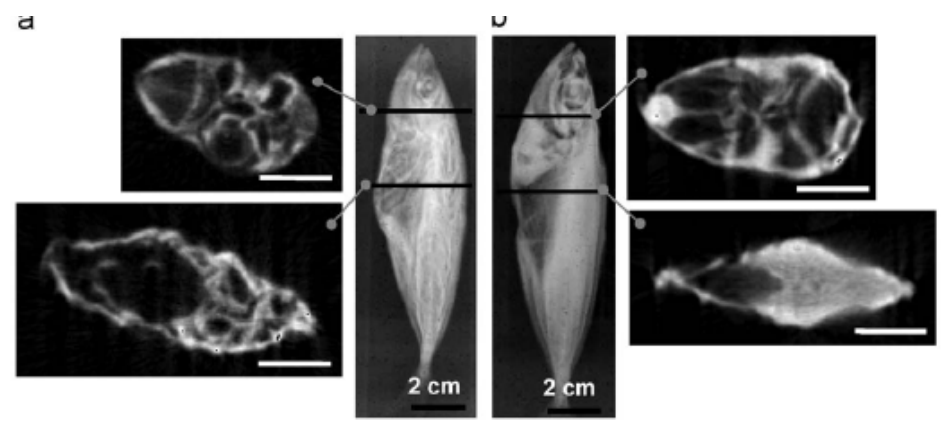

Figure 10. Neutron images of fish (center) and reconstructed tomography slices (one at the level of the head of the fish and another at the level of the shoulder) recorded on mackerel treated by freeze drying (a- left) and by conventional drying (soft breeze) (b- right) [28].

\subsubsection{Cooking of meat}

Cooking meat modifies its structure resulting in the improvement of its taste, aroma and texture. In fact, most of the biochemical reactions which are responsive of these changes are linked to the proteins which constitute meat: myosin, heamprotein and collagen. The changes in the meat microstructure and the concomitant juice release have been recently observed by neutron imaging [29]. The analysis of the attenuation cartography (as presented in Figure 11) realized on images collected during cooking in a semi-professional oven (placed in front of the neutron detector) highlights the fact that the main microscopic shrinkage of the meat happens after $70^{\circ} \mathrm{C}$ (after myosin and collagen denaturation). More important changes of attenuation have been observed at higher temperature (around $80^{\circ} \mathrm{C}$ ) and are related to juice release following the protein denaturation.
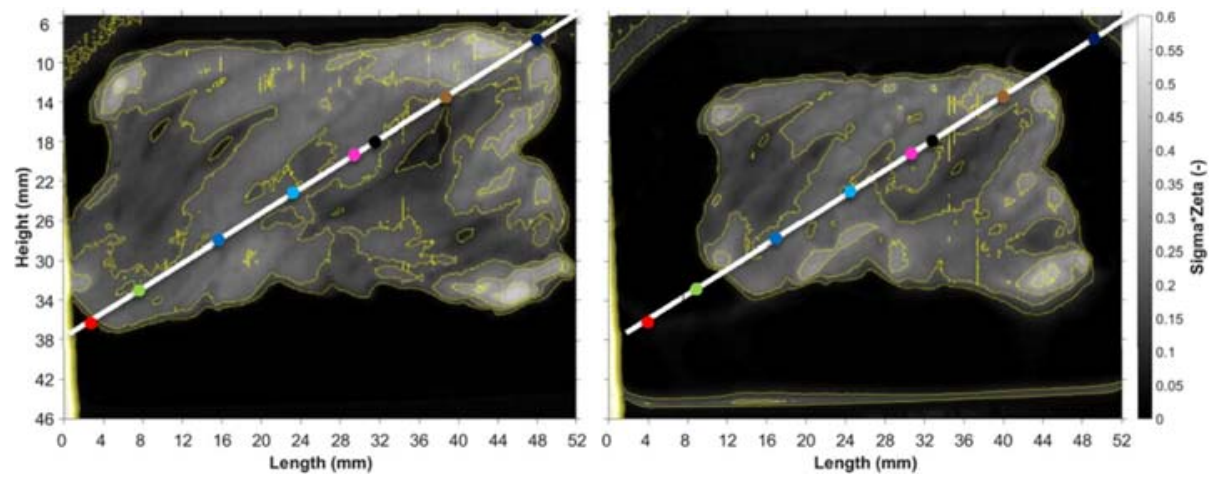

Figure 11. Cartographies of beef meat slices: raw (left) and cooked at $106^{\circ} \mathrm{C}$ (right) [29].

In this study, the process of cooking in the oven has been recapitulated in the neutron beam. The main goal was to better determine the key temperature to observe morphological changes due to contraction of the meat fibres caused by cooking and migration of water (juice) inside the sample and towards the outside. 


\subsection{Imaging structure and defects of food and packaging}

\subsubsection{Water loss in fruit}

During storage and shelf life, fruit loose water which reduces their weight and causes the decrease of the organoleptic quality and acceptance by the consumer. A multiphysics model was applied to study the dehydration of apple tissue accompanied by large mechanical deformation [30]. The model incorporated a water transport model and a nonlinear viscoelastic deformation model. To visualize and quantify the water transport in apple tissue neutron imaging and tomography were performed on cylindrical samples of apples (diameter $11 \mathrm{~mm}$ and height $20 \mathrm{~mm}$ ). Dehydration of these cylindrical apple tissues has also been studied by gravimetrical measurements and by numerical modelisation. As shown in Figure 12, the experimental mass loss results (neutron and gravimetrical) and the predicted results are in agreement.

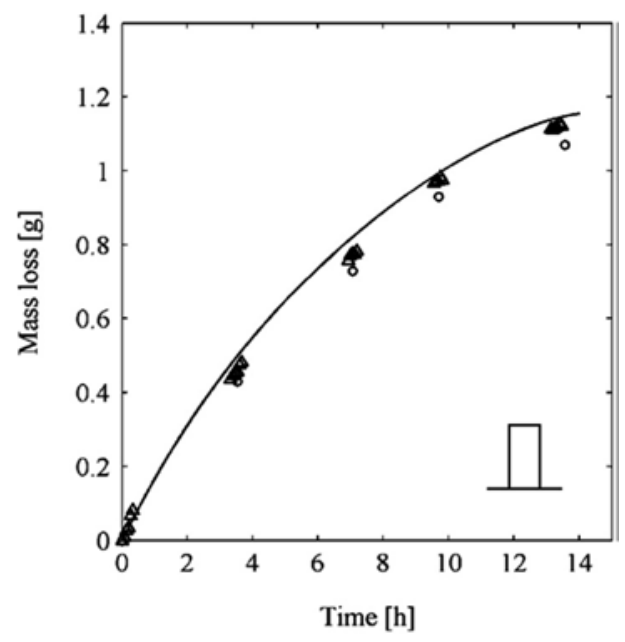

Figure 12. Water loss from apple tissue overtime: balance (o), radiography $(\Delta)$ and model (solid line) [30].

The accuracy of the model was checked more in detail by comparing the predicted local water distribution with those from the neutron tomography. In addition to moisture content, also shrinkage predicted by numerical model was compared with experiments by neutron to further validate the model as shown in Figure 13. 
(a)

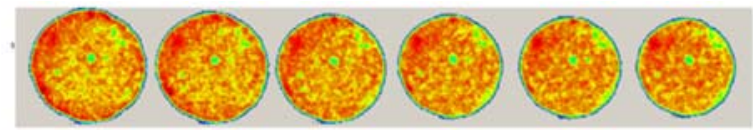

(b)

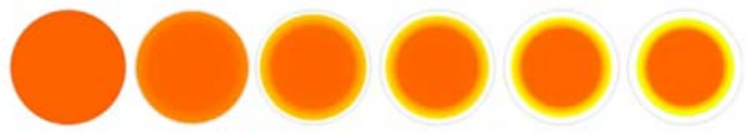

(c)

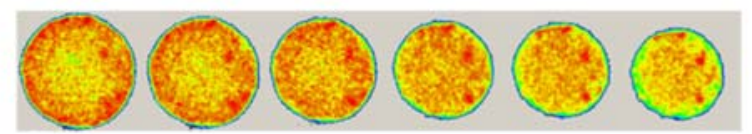

(d)

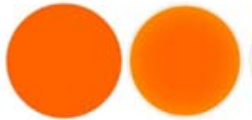

Oh

$1 \mathrm{~h}$

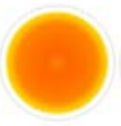

$3 \mathrm{~h}$

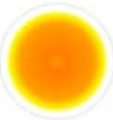

$4 \mathrm{~h}$

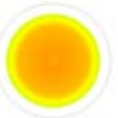

$5 \mathrm{~h}$

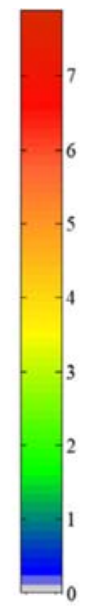

$6 \mathrm{~h} \quad X_{-} W_{d b}\left(\mathrm{~kg} \mathrm{~kg}_{d m}^{-1}\right)$

Figure 13. Horizontal slices of water content through the apple tissue as function of time. (a) Reconstructed neutron tomography image at the middle layer of the sample $(\mathrm{y}=10 \mathrm{~mm})$. (b) Numerical model at the middle layer. (c) Reconstructed neutron tomography iamge near the top layer $(y=17.5 \mathrm{~mm})$. (d) Numerical model near the top layer $(17.5 \mathrm{~mm})$. (X_Wdb: dry base water content)[30].

Neutron tomography revealed the heterogeneity of the water distribution of apple tissue. The total water loss and local water content obtained by experiments and simulations are comparable, indicating the numerical model developed in this study is a viable alternative to neutron.

\subsubsection{Structural analysis of dried corn kernels}

Neutron tomography was used for the structural analysis of dried corn kernels. The analysis of the image (Figure 14) obtained by neutron tomography showed that this technique was able to identify well-known internal structure of corn kernel. Many structures visible in the corn kernel reconstructions were identifiable as well-known anatomical features of corn kernels as shown in Figure 14. 


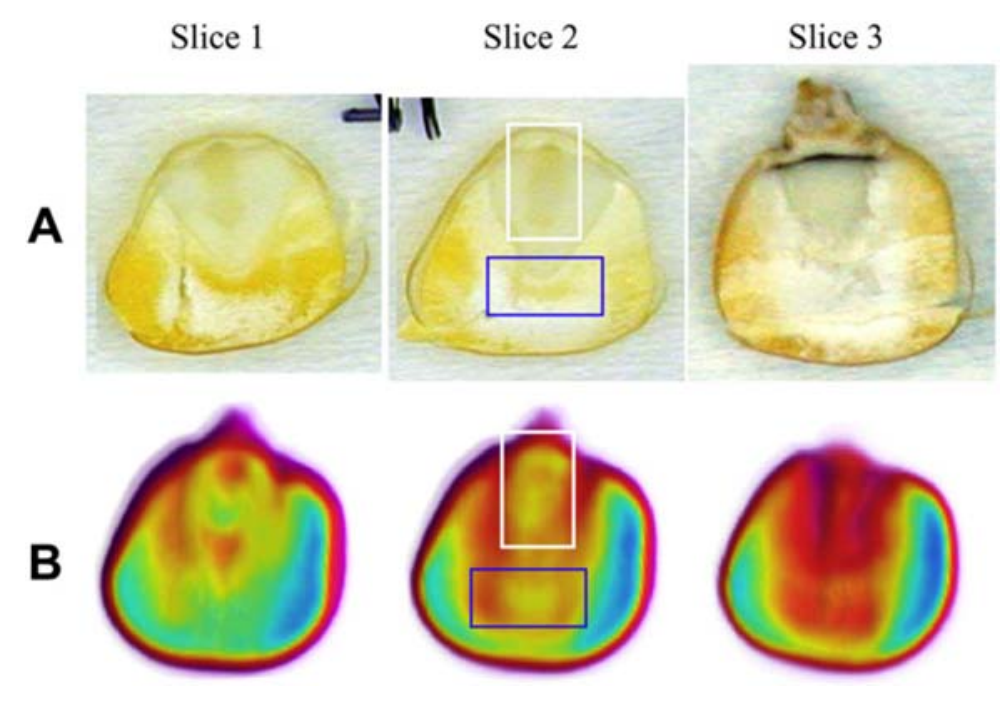

Figure 14. Reconstructed (B) vs. physical kernel slices (A) [31].

The main goal of this study [31] was to determine if these un-destructive experiments can discriminate different type of treatment of kernels. Indeed, kernels which were infected, or not, by aflatoxin producing fungus Aspergillus flavus were analyzed. Two varieties of kernel were used, one susceptible to fungus infection and one resistant. Infected kernels were found to have a lower neutron attenuation in some specific part of their anatomical structure (scutellum and embryo regions) possibly caused by lower hydrogen concentrations due to fungal degradation.

\subsubsection{Defect in corks stoppers}

Cork is used in a variety of products going from construction materials to gaskets, but its most important use is as a stopper for premium wines. The first known use of cork as a closure dates back to the fifth century BC, when it was used with Greek amphora. Nevertheless, the rise of cork started at the fifteenth century with the beginning of glass wine bottles. The principle requirements for cork stoppers are the homogeneity of the cork and the lack of cavities / and or cracks. Cork commonly used for wine stoppers comes from the bark of the oak tree Quercus suber L. The quality grading of cork stoppers is based on visual analysis of transverse and tangential sections of cork planks, taking into account the three main types of defects: pores (lenticular channels), physiological anomalies (nails, clay), pathogenic anomalies (insect galleries). Whatever the technique used, cork stoppers are grading in different classes. Generally there are 7 or 8 qualities [32]. Class 0 represents the best quality while 6 or 7 (depending on the classification used) is the poorest. As presented in Figure 15, the defects of cork stoppers from two classes ( 0 and 4$)$ were analyzed by neutron imaging and tomography [33]. 
a

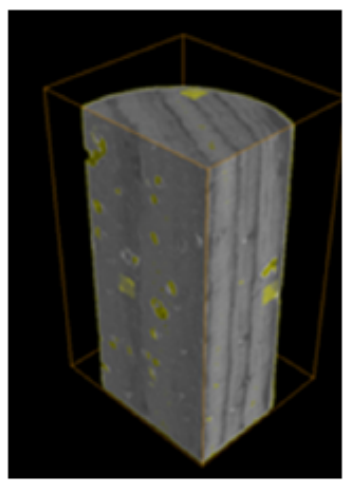

b

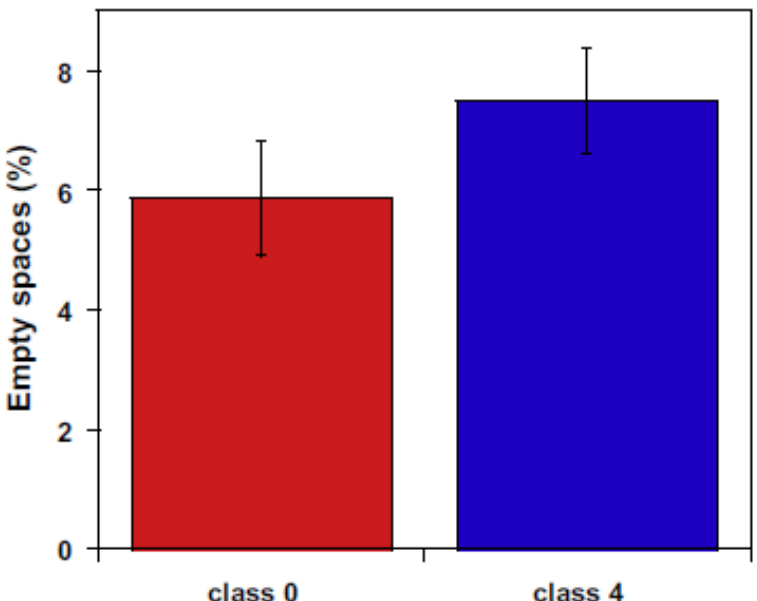

Figure 15. Analysis of defects in cork stoppers by neutron tomography. a) Visualization in 3 dimensions of a natural cork stopper (grade 0 ) obtained by neutron tomography; b) Histogram representing the differences of empty space content between natural corks from grade 0 and grade 4 [33].

Surface analysis of defects obtained from photography leads to more surface defects in class 4 than in class 0 . Neutron radiography and tomography are powerful methods that really show the defects inside the material. Moreover, tomography also allows observing defects distribution along the whole stopper and possible interconnectivity.

\section{Conclusion}

Neutron imaging and tomography are more and more used for high spatial (resolution of few micrometers on large objects) and temporal (few second to obtain a 2D image and few hours to obtain $3 \mathrm{D}$ reconstruction) characterization of the microstructure of food, packaging or in agronomy. The main advantages of the neutron arise from the fact that they are selective (mainly to hydrogen), they can penetrate deep level of aluminium, so studies can be performed in complex surrounding sample, and they are non-destructive. Recently neutron imaging (and tomography) has been used to study the impact of process (temperature, pressure, drying...) in -situ and determine the change of structure of the food as function of this process. Thanks to the high selectivity of neutron, moreover through their high sensitivity to hydrogen (compared to deuterium), the migration of water in food or in vegetable, or leave and plants by neutron imaging experiments is more and more studied. These experiments allow to better understand the crucial role of water, hydration on microstructural properties of food matrices and moreover their link to organoleptic properties (such as texture, color etc..).

\section{References}

1. B. Schillinger, E. Lehmann, P. Vontobel, Physica B, 59, 276 (2000)

2. N. Kardjilov, A. Hilger, I. Manke, M. Strobl, W. Treimer, J. Banhart, Nuclear Instruments and Methods In Physics Research Section A, 542, 16 (2005)

3. I.S. Anderson, R. McGreevy, H. Bilheux, Neutron Imaging and Applications: A Reference for the imaging Community, (Springer, New York, 2009) 
4. M. Dierick, J. Vlassenbroeck, B. Masschaele, V. Cnudde, L. Van Hoorebecke, A. Hillenbach, Nuclear Instruments and Methods In Physics Research Section A, 542, 296 (2005)

5. B. Schillinger, H. Abele, J. Brunner, G. Frei, R. Gähler, A. Gildemeister, A. Hillenbach, E. Lehmann, P. Vontobel, Nuclear Instruments and Methods in Physics Research A, 542, 142 (2005)

6. N. Takenaka, T. Kadowaki, Y. Kawabata, I.C. Lim, C.M. Sim, Nuclear Instruments and Methods in Physics Research A, 542, 129 (2005)

7. M. Kang, H.Z. Bilheux, S. Voisin, C.L. Cheng, E. Perfect, J. Horita, J.M. Warren, Nuclear Instruments and Methods In Physics Research Section A, 708, 24 (2013)

8. S. Deabate, G. Gebel, P. Huguet, A. Morin, G. Pourcelly, Energy Environ. Sci., 5, 8824 (2012)

9. M. Klages, S. Enz, H. Markotter, I. Manke, N. Kardjilov, J. Scholta, Journal of Power Sources, 239, 596 (2013)

10. K., Ryzewski, S. Herringer, H. Bilheux, L. Walker, B. Sheldon, S. Voisin, J-C Bilheux, V. Finoocchiaro, Physics Procedia, 43, 343 (2013)

11. D. Mannes, F. Schmid, J. Frey, K. Schmidt-Ott, E. Lehmann, Physics Procedia, 69, 653 (2015)

12. J. Teixeira, R. Magli, C. Loupiac, European Journal of Mineralogy, 27 (3), 289 (2015)

13. D. Mannes, E. Lehmann, P. Cherubini, P. Niemz, Trees, 21, 605 (2007)

14. M. Sedighi-Gilani, M. Griffa, D. Mannes, E. Lehmann, J. Carmeliet, International Journal of Heat and Mass Transfer, 55, 6211 (2012)

15. P. Boillat, G. frei, E.H. Lehmann, G.G. Scherer, A. Wokaum, Electrochem. Solid-State Letters, 13, B25 (2010)

16. E. H. Lehmann, A. tremsin, C. Grunzweig, I. Johnson, P. Boillat, L. Josic, Journal of Instrumentation, 6, C01050 (2011)

17. E.H. Lehmann, P. Vontobel, G. Frei, G. Kuehne, A. Kaestner, Nuclear Instruments and Methods In Physics Research Section A, 651, 1 (2011)

18. N. Kardjilov, M. Dawson, A. Hilger, I. Manke, M. Strobl, D. Penumadu, F.H. Kim, F. GarciaMoreno, J. Banhart, Nuclear Instruments and Methods In Physics Research Section A, 651, 95 (2011)

19. N. Kardjilov, A. Hilger, M. Dawson, I. Manke, J. Banhart, M. Strobl, P. Boni, Journal of Applied Physics, 108, 034905 (2010)

20. A.S. Tremsin, J.V. Vallerga, J.B. McPhate, O.H.W. Siegmund, W.B. Feller, L. Crow, R.G. Cooper, Nuclear Instruments and Methods in Physics Research A, 592, 374 (2008)

21. A.S. Tremsin, J.B. McPhate, J.V. Vallerga, O.H.W. Siegmund, J.S. Hull, W.B Feller, E. Lehmann, Nuclear Instruments and Methods in Physics Research A, 604, 140 (2009)

22. A.S. Tremsin, J.B. McPhate, J.V. Vallerga, O.H.W. Siegmund, W.B. Feller, E. Lehmann, L.G. Butler, M. Dawson, Nuclear Instruments and Methods In Physics Research Section A, 652, 400 (2011)

23. A.S. Tremsin, W.B. Feller, R.G. Downing, Nuclear Instruments and Methods in Physics Research A, 539, 278 (2005)

24. M. Estribeau, P. Magnan, Proceedings of SPIE, 5251, 243(2004)

25. F. Kharfi, O. Denden, A. Bourenane, T. Bitam, A. Ali, Applied Radiation And Isotopes, 70, $162(2012)$

26. S.H. Williams, A. Hilger, N. Kardjilov, I. Manke, M. Strobl, P.A. Douissard, T. Martin, H. Riesemeier, J. Banhart, Journal of instrumentation, 7, P02014 (2012)

27. C. Grünzweig, G. frei, E. Lehman, G. Kühne, C. David, Review of Scientific Instruments, 78, 053708 (2007)

28. K. Tanoi, Y. Hamada, S. Seyama, T. saito, H. Likura, T. M. Nakanishi, Nuclear Instruments and Methods in Physics Research A, 605, 179 (2009)

29. S. Scussat, F. Ott, A. Hélary, S. Desert, P. Cayot, C. Loupiac, Food Biophysics, 11, 207 (2016)

30. W. Aregawi, T. Defraeye, S. Saneinejad, P. Vontobel, E. Lehmann, J. Carmeliet, D. Derome, P. Verboven, B. Nicolai, Int. J. Heat and Mass Transfer, 67, 173 (2013) 
31. 31. T.E. Cleveland IV, D.S. Hussey, Z-Y. Chen, D.L. Jacobson, R.L. Brown, C. CarterWientjes, T.E. Cleveland, M. Arif, J. of Cereal Science, 48, 517 (2008)

32. A. Ferreira, F. Lopes, H. Pereira, Annals of Forest Science, 57(2) 187 (2000)

33. A. Lagorce-tachon, T. Karbowiak, C. Loupiac, A. Gaudry, F. Ott, C. Alba-Simionesco, R. Gougeon, V. Alcantara, D. Mannes, A. Kaestner, E. Lehmann, J-P. Bellat, Journal of Food Engineering, 149, 214 (2015) 